\title{
Efficacy and adverse effects of intravenous lignocaine therapy in fibromyalgia syndrome
}

\author{
JH Raphael*1, JL Southall ${ }^{1}$, GJ Treharne ${ }^{2}$ and GD Kitas ${ }^{3}$
}

Address: ${ }^{1}$ Department of Pain Management, Dudley Group of Hospitals NHS Trust, West Midlands, UK, ${ }^{2}$ School of Psychology, University of Birmingham, Edgbaston, Birmingham, UK and ${ }^{3}$ Department of Rheumatology, Dudley Group of Hospitals NHS Trust, West Midlands, UK

E-mail: JH Raphael* - JonRaph@AOL.com; JL Southall - julie.burrows@dudleygoh-tr.wmids.nhs.uk; GJ Treharne - GJ884@psg-fs4.bham.ac.uk; GD Kitas - g.d.kitas@bham.ac.uk

*Corresponding author

Published: 8 September 2002

BMC Musculoskeletal Disorders 2002, 3:21

This article is available from: http://www.biomedcentral.com/I47/-2474/3/2I

(C) 2002 Raphael et al; licensee BioMed Central Ltd. This article is published in Open Access: verbatim copying and redistribution of this article are permitted in all media for any non-commercial purpose, provided this notice is preserved along with the article's original URL.

Keywords: Fibromyalgia, Lidocaine, Intravenous, Adverse Effects, Side-Effects, Benefits, Pain, Psychosocial, Depression, Quality of Life

\begin{abstract}
Background: To investigate the effects of intravenous lignocaine infusions (IV lignocaine) in fibromyalgia.

Methods: Prospective study of the adverse effects of IV lignocaine in 106 patients with fibromyalgia; retrospective questionnaire study of the efficacy of IV lignocaine in 50 patients with fibromyalgia.

Results: Prospective study: Two major (pulmonary oedema and supraventricular tachycardia) and 42 minor side-effects were reported. None had long-term sequelae. The commonest was hypotension (17 cases). Retrospective study: Pain and a range of psychosocial measures (on single II-point scales) improved significantly after treatment. There was no effect of the treatment on work status. The average duration of pain relief after the 6-day course of treatment was II.5 \pm 6.5 weeks.
\end{abstract}

Conclusions: Intravenous lignocaine appears to be both safe and of benefit in improving pain and quality of life for patients with fibromyalgia. This needs to be confirmed in prospective randomised controlled trials.

\section{Background}

Fibromyalgia is a chronic syndrome with widespread continuous muscular pain at rest and tenderness to pressure in at least 11 of 18 specified anatomical sites [1]. It is a common condition with a prevalence in the adult population of 2\% [2] and causes considerable disability equivalent to that seen in rheumatoid arthritis [3]. Chronicity is the rule and disability and depression increase with disease duration [4].
Treatments fall into several classes: pharmacological, psychological, physical and complementary therapies. Nonsteroidal anti-inflammatory drugs and steroids are unhelpful [5,6]; tricyclic antidepressants are widely used but their effect is modest $[7,8]$. Cognitive behavioural therapies may be useful but are difficult to assess in controlled settings [9]. Electroacupuncture provides only short-term benefit [10], while cardiovascular conditioning has not 
been adequately studied [11-13]. Clearly an effective treatment is lacking.

Fibromyalgia is poorly understood in neurobiological terms with no general agreement about its aetiology and pathogenesis. Some authorities contend it is a learned behavioural response to chronic stress [14], whereas others view it in terms of a neuroendocrine abnormality [15]. These two proposed mechanisms may each lead to the other and their manifestations may co-exist.

The symmetry of the condition leads to the hypothesis of a central nervous system disturbance [16]. Pharmacological interference with central pain processing can be achieved in two ways: either by augmenting the action of inhibitory pain pathways or by inhibiting the action of pain pathways. Inhibitory pathway augmentation may be achieved by noradrenergic and serotonergic drugs or by opioids. Pain pathway inhibition may be achieved by systemic administration of local anaesthetics through their inhibition of voltage-gated sodium channels. The effectiveness of the latter, particularly of IV lignocaine, in neuropathic conditions is well established $[17,18]$. However, the use of IV lignocaine in fibromyalgia has not been investigated extensively. The effect of a single infusion of 5 $\mathrm{mg} / \mathrm{kg}$ was investigated in a small number of patients by Sorenson [19]. This demonstrated significant pain relief in some patients for up to five days. A previous open study from our unit evaluated daily lignocaine infusions of 5-7 $\mathrm{mg} / \mathrm{kg}$ given over 6 days to a small sample of 10 patients [20]; this showed benefit in pain relief and mood scores at 30 day follow up, it did not address duration of pain relief, other quality of life indicators or adverse effects.

The aims of the present studies were (a) to document and quantify any adverse effects of intravenous lignocaine therapy for fibromyalgia patients; (b) to determine if this therapy has benefit upon pain and quality of life; and (c) to measure the duration of benefit.

\section{Methods}

\section{Description of therapy}

The lignocaine intravenous infusional therapy was only given to patients with evidence of normal electrocardiographic conduction and normal serum electrolyte concentrations to minimise risks of cardiac dysrhythmias.

Patients had continuous electrocardiographic monitoring and measurements of pulse and blood pressure every 15 minutes by automated device during infusion. They were kept under close observation by nursing staff taught to detect symptoms and signs of neurological side-effects such as tinnitus and tunnel vision.
Serial infusions of intravenous lignocaine were given for six consecutive days. They were started at $5 \mathrm{mg} / \mathrm{kg}$ minus $100 \mathrm{mg}$ and increased by $50 \mathrm{mg}$ per day to $5 \mathrm{mg} / \mathrm{kg}$ plus $150 \mathrm{mg}$, provided the maximum was no greater than 550 mg. The lignocaine was infused in Hartman's solution $500 \mathrm{mls}$ over 6 hours. The infusion rate was reduced if there was bradycardia, tachycardia, hypotension, headaches, dizziness, arrhythmias or tunnel vision. If slowing the infusion failed to correct these then the infusion was stopped.

\section{Patients}

Patients were recruited sequentially from those with fibromyalgia [1] scheduled for intravenous lignocaine therapy who had not achieved sufficient symptom relief using more "traditional" modes of treatment either in the rheumatology or pain relief departments. No patient was treated more than once in the study period. Adverse effects were assessed in a prospective study of patients with fibromyalgia having IV lignocaine during a six-month period this yielding just over 100 patients, a number thought likely to provide useful information on adverse events. The patients' nurse was requested to complete a questionnaire addressing any side-effects noted at the time of their treatment. These adverse effects were later computed by a third party not working on the ward. From 110 consecutive patients, $106(96 \%)$ usable questionnaires were returned. The mean (range) age of this sample was 51.4 (29-74) years; 88 of the 106 (92\%) were female; 72 $(75 \%)$ had primary fibromyalgia, the remainder had fibromyalgia secondary to osteoarthritis.

Efficacy was assessed retrospectively by sending postal questionnaires during a different time period to another sample of patients who had sequentially received this therapy until 50 usable replies were obtained, this occurred after sending out 55 questionnaires ( $91 \%$ response rate). The smaller number was chosen because of the greater work involved in this more lengthy questionnaire. There was an overlap between the two samples with 29 $(58 \%)$ of those investigated for efficacy being included in the study of adverse effects. The age range of the 50 responders was 28-73 years with a mean of $50.2 \pm 10.0 ; 40$ $(80 \%)$ were female; $42(84 \%)$ had primary fibromyalgia, the remainder had fibromyalgia secondary to osteoarthritis. The mean duration of fibromyalgia was $6.6 \pm 4.5$ years with a range of 1-19.

The postal questionnaire was anonymised and requested demographic data (including duration of disease); ratings of pain and a range of psychosocial aspects on 11-point numerical rating scales $[21,22]$; and comments on use of analgesics, General Practitioner (GP) visits and satisfaction from treatment. Scoring was requested for symptoms both prior to and following intravenous lignocaine as re- 
Table I: Comparison of Ist and multiple IV lignocaine treatment groups

\begin{tabular}{|c|c|c|c|}
\hline & Ist time IV lignocaine treatment & multiple IV lignocaine treatments & statistical analysis \\
\hline Number & 13 & 37 & \\
\hline age (mean, SD) & $54.3(4.0)$ & $49.6(10.7)$ & $t(44)=-0.93, N S$ \\
\hline $\operatorname{sex}(f: m)$ & $12: 1$ & $28: 9$ & $\chi^{2}(I, N=50)=0.10, N S$ \\
\hline Duration of FMS, yrs, mean (SD) & $5.7(5.3)$ & $6.8(4.3)$ & $\mathrm{t}(42)=-0.11, \mathrm{NS}$ \\
\hline pre-treatment hrs in pain (mean, SD) & $23.0(2.2)$ & $21.8(4.5)$ & $t(42)=-0.83, N S$ \\
\hline co-morbidity & 5 & 9 & $\chi^{2}(I, N=50)=2.87, \mathrm{NS}$ \\
\hline
\end{tabular}

Table 2: Side-effects of IV lignocaine $(n=106)$

\begin{tabular}{lc}
\hline Side-Effect & Number of Incidents(\%) \\
\hline Hypotension & \\
Hypertension & $17(16)$ \\
Tachycardia & $5(5)$ \\
Arrythmia (SVT) & $\mathrm{I}(1)$ \\
Pulmonary Oedema & $\mathrm{I}(\mathrm{I})$ \\
Headache & $\mathrm{I}(\mathrm{I})$ \\
Nausea & $8(8)$ \\
Abdominal Pain & $\mathrm{I}(\mathrm{I})$ \\
Increased Joint Pain & $\mathrm{I}(1)$ \\
Chest Pain & $\mathrm{I}(1)$ \\
Miscellaneous & $\mathrm{I}(\mathrm{I})$ \\
& $7(7)$ \\
\hline
\end{tabular}

called by the patients. Patients who had undergone this therapy more than once were asked to answer the questionnaire in relation to the most recent course of treatment. Of the patients who responded, this applied in 37 of the 50 cases $(74 \%)$; for the remainder, only one treatment had been received. Demographic and clinical data on these two groups is presented in Table 1.

\section{Pain measuring}

Pain was measured by pain score on the 11-point numerical rating scale (where 0 represents no pain and 10 represents maximal imaginable pain), a four-point verbal scale of pain severity (none, mild, moderate, severe) and average hours per day in pain. Pain relief was measured on the 11 -point numerical rating scale (where 0 represents maximal imaginable relief and 10 represents no relief). Duration of pain improvement after treatment, and the perceived worthiness of the treatment was also requested.

\section{Measurement of depression, coping ability and dependen- cy}

The psychological dimension of the pain and its relief was addressed by measurement of depression, coping ability and dependency. These questions used the 11-point scales with 0 to represent no depression, no dependency or no interference with coping ability due to pain and 10 to represent maximal imaginable depression, dependency or maximal interference with coping ability due to pain. The sociological dimension of the pain and its relief was addressed by measurement of sleep, social life, work, housework, mobility, driving and sex life. These questions used the 11-point scales with 0 to represent no interference in the activity due to pain and 10 represented the maximum interference imaginable in the activity due to pain.

The utilisation of healthcare resources was addressed by measurement of patient visits to their GP before and after treatment.

\section{Statistical analyses}

Adverse effects were analysed by frequency of occurrence. Relationships between the aspects of the benefits of treatment were analysed by Spearman's non-parametric correlations. Group differences on the benefits were analysed using chi-squared tests, Mann-Whitney U-tests and independent samples t-tests, as appropriate; differences between pre- and post-treatment scores were analysed using chi-squared tests, Wilcoxon signed ranks tests, and paired samples t-tests. To account for multiple tests, Bonferroni correction was applied and significance determined at $\mathrm{p}<$ 0.005 .

\section{Results \\ Side-effects}

There were 44 documented adverse effects (42\%) (Table 2 ), of which two were serious (2\%). The commonest sideeffect was hypotension (requiring slowing or stopping infusion temporarily, but none requiring other active measures). The most serious were a supraventricular tachycardia requiring pharmacological treatment and a case of pulmonary oedema requiring diuresis. In both of these cases the infusion was aborted. The miscellaneous group included infected cannula site, tissued infusion and reaction to ECG electrode pads. None had long-term sequelae. 


\section{Fig 1. Pain severity pre- and post- lignocaine}

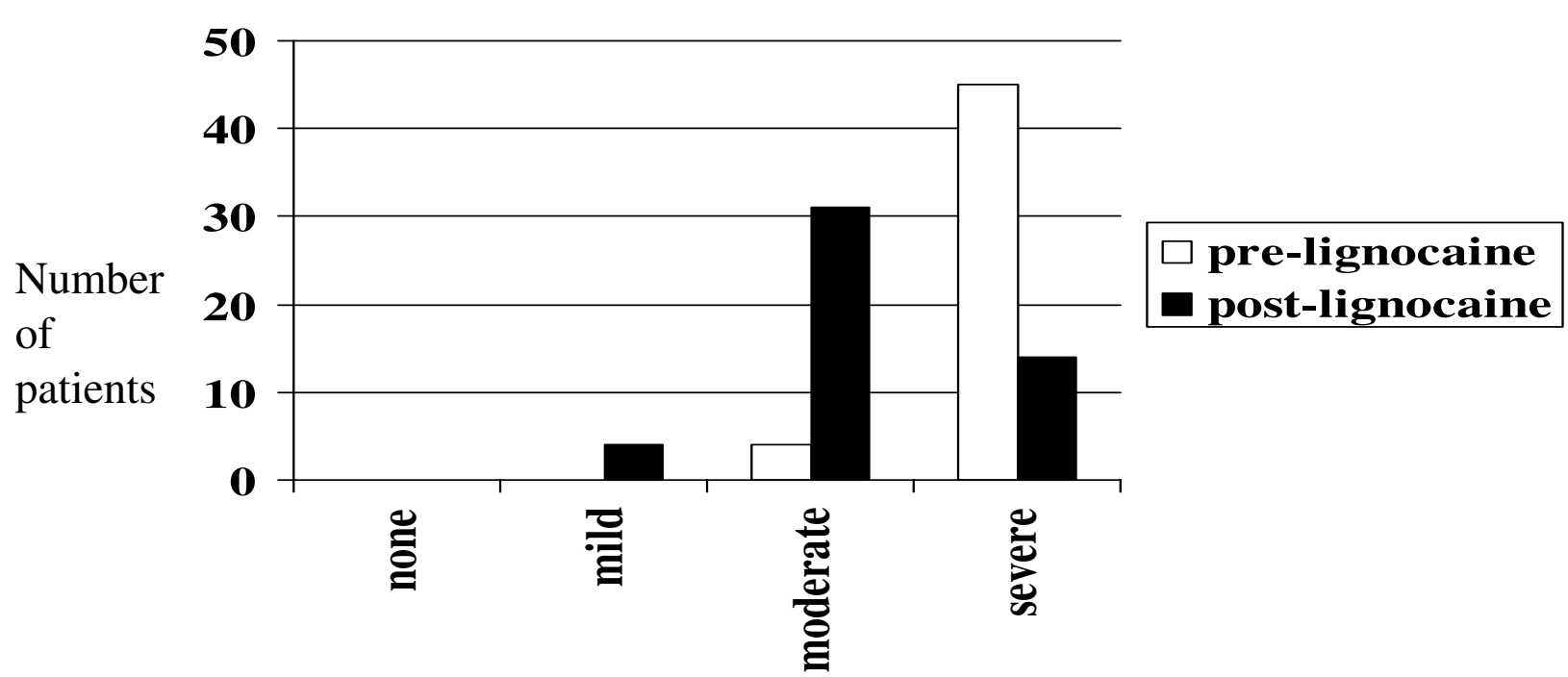

Figure I

Pain severity pre-and post-lignocaine

Table 3: Efficacy of IV lignocaine in fibromyalgia

\begin{tabular}{|c|c|c|c|c|c|}
\hline Dimension & Variable & Pre-Treatment Average ${ }^{\mathrm{a}}$ & Post-Treatment Average & Test Statistic ${ }^{b}$ & $P$ \\
\hline \multirow[t]{3}{*}{ Sensory } & Pain & 9 & 5 & 5.74 & $<0.001$ \\
\hline & Pain Relief & 9 & 5 & 5.11 & $<0.001$ \\
\hline & Hours/Day in Pain & 22.97 & 17.42 & 4.27 & $<0.001$ \\
\hline \multirow[t]{4}{*}{ Psychological } & Depression & 8 & 5 & 4.32 & $<0.001$ \\
\hline & Quality of life & 9 & 6 & 4.94 & $<0.001$ \\
\hline & Coping & 9 & 5 & 5.45 & $<0.001$ \\
\hline & Dependency & 8 & 5 & 3.70 & $<0.001$ \\
\hline \multirow[t]{7}{*}{ Sociological } & Sleep & 9 & 5 & 5.20 & $<0.001$ \\
\hline & Work & 10 & 10 & 2.63 & NS \\
\hline & Social Life & 10 & 7 & 4.81 & $<0.001$ \\
\hline & Sex Life & 9 & 7.5 & 3.36 & $<0.001$ \\
\hline & Driving & 6.5 & 4.5 & 3.77 & $<0.001$ \\
\hline & Housework & 8 & 7 & 4.18 & $<0.001$ \\
\hline & Mobility & 9 & 7 & 4.22 & $<0.001$ \\
\hline
\end{tabular}

a The reported average is the median for all variables, except in the case of Hours/Day in Pain, where the mean is given. $b$ The reported statistic is the $\mathrm{Z}$ score converted from the Wilcoxon signed ranks test for all variables, except in the case of Hours/Day in Pain, where the $t$ statistic is given.

\section{Benefits}

Overall, the treatment was considered to be worthwhile by the patients, with 32 (2/3 of the valid 48 responses to this question) responding that it was very worthwhile, 6 $(1 / 8)$ responding that it was quite worthwhile, 6 respond- ing that it was adequate and only $4(8 \%)$ responding that it was not worthwhile.

Pain score, pain relief interruption, mean daily duration of pain and verbal assessment of pain all reduced signifi- 


\section{Fig 2. Duration of pain relief with IV lignocaine}

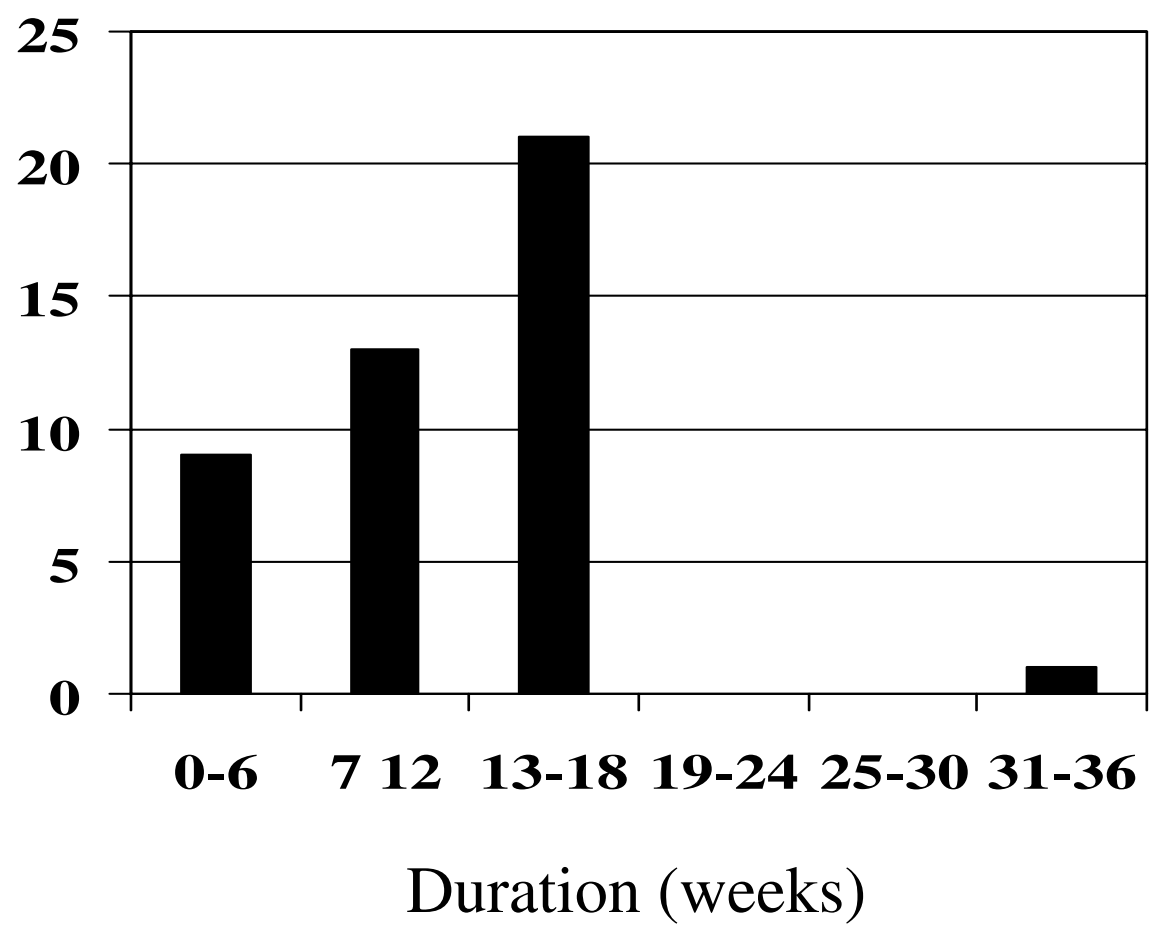

Figure 2

Duration of pain relief with IV lignocaine

cantly (table 3 and figure 1). Verbal assessment of pain most commonly changed from severe to moderate (figure 1 ), and when these ratings were converted to a 4-point numerical system, the improvement was significant (Wilcoxon $\mathrm{W}=630, \mathrm{Z}=5.71, \mathrm{P}<0.001)$, and the relationship with the 11-point pain score was moderate to strong ( $\rho=$ 0.44 for pre-treatment scores and 0.54 for post-treatment scores, both $\mathrm{P}<0.001)$. The mean duration of pain relief was $11.5 \pm 6.5$ weeks, range $0-36$ weeks (Figure 2).

No differences existed between these first-time and multiple-treatment patients. $\mathrm{t}(36)=0.29$, NS for post-treatment hours of pain per day; $\mathrm{t}(43)=-1.18$, NS, for the duration of relief; and Mann-Whitney $U$ ranged from 239.50 to $129.00, \mathrm{Z}$ ranged from 1.36 to 0.05 , all NS, for the nonparametric pre- and post-treatment pain and psychosocial variables.

Analyses of the Spearman's correlations of all pain and psychosocial questionnaire items indicate that on average they were only moderately related, with a mean $\rho$ of 0.44 (within the same time of reference), from a range of 0.00 (NS) $-0.87(\mathrm{P}<0.001)$. However, to avoid error due to multiple measures, Bonferroni correction was applied with significance determined by $\mathrm{p}<0.005$.

Across all analyses of pre- and post-treatment scores, beneficial change was seen in the majority of cases on most variables: the median number of detrimental changes per variable was 2 , with a range of $0-5$. Tied scores were only moderately common, with a median number of $15(<1 /$ 3 ) per variable, from a range of 6-28.

Psychological and sociological dimensions of pain experience were significantly improved across all domains apart from the effect of pain upon work status (Table 3 ).

The median number GP visits per month reduced from 1.5 to 0.5 after treatment (Wilcoxon $\mathrm{W}=344, \mathrm{Z}=3.73, \mathrm{P}$ $<0.001)$. 


\section{Discussion}

Fibromyalgia is a chronic syndrome with a variety of symptoms including widespread muscular pain, tenderness, unrefreshed sleep, fatigue and is frequently associated with irritable bowel and bladder symptoms, as well as disability, reduced quality of life and depression [4]. It is defined in terms of pain and tenderness [1] supporting the notion that fibromyalgia is a pain disorder.

Systemic local anaesthetics are primarily prescribed for their antiarrhythmic actions. Short term analgesia is well documented with intravenous lignocaine in a variety of neuropathic pain states such as diabetes [17] and postherpetic neuralgia [18], and some of these patients appear to derive prolonged relief from such treatment [23].

The mechanism of local anaesthetic infusional therapy is conjectural. There is evidence for disordered sensory processing both at a peripheral and at a central level in fibromyalgia. Sorenson [24] showed by injecting intramuscular saline that there was experimentally induced hyperexcitability in fibromyalgia patients compared with controls. Gibson [25] described hyper-responsive somatosensory evoked potentials (SSEP) to laser skin stimulation and Lorenz [26] showed there was a response to SSEP in both hemispheres compared to controls. The reduced thalamic blood flow seen on SPECT imaging [27] is similar to that encountered in unilateral chronic neuropathic pain using PET [28]. With evidence for disordered pain processing centrally and peripherally in fibromyalgia one can consider it a neuropathic condition providing a rationale for the use of systemic local anaesthetics.

Our data are similar to the other two studies of intravenous lignocaine $[19,20]$. Both of these found significant pain relief after treatment, and one of these which measured mood found that this also improved [20]. Other dimensions of pain experience were not measured in these studies.

In the present study the magnitude of effect for pain relief was a 4-point reduction on an 11-point scale; this is likely to be clinically significant. It also fell outside two standard deviations of the average after treatment compared with before, meeting quantitative tests on statistical versus clinical significance [29]. The duration of pain relief averaging 11.5 weeks is also clinically significant; however, the reduction in the number of hours per day in pain fell by only 5 of the 24 hours in the day and suggests that this therapy reduces the severity of pain rather than giving significantly longer pain free periods.

A range of moderately related quality of life measures, that included the psychological and sociological dimension of pain experience, were significantly improved after treat- ment. Notably, depression, coping, sleep, social life and mobility all improved. The effect of pain upon work did not change. This situation may reflect the socio-economic factors that affect work status which are beyond therapeutic control.

Most of the patients who were sent questionnaires had received this treatment before (37 of the 50 usable replies). It is our clinical practice to not consider repeating this therapy if two sequential treatments are considered ineffective by the patient, thus one might expect a higher proportion of responders to the treatment compared with an unselected group of fibromyalgia patients. However, the results were similar in those who had received this treatment on more than one occasion and in those who had only had the treatment once although the sample for whom this was first treatment was small $(n=13)$.

The retrospective design used relies upon patient recall. Patients can overrate past pain [30] and thus overestimate benefits of a treatment; however, overestimates seem to particularly occur when pain measures have increased over the treatment period, which was not the case in this study [31]. Pain was our primary endpoint and as a subjective experience is difficult to measure. Rating scales are commonly used for their simplicity and the high associated response rate. We chose to use a numerical rating scale as this compares favourably against visual analogue and verbal rating scales in terms of minimising scoring errors and optimising sensitivity [21].

We included in our questionnaire a range of single questions to cover the dimensions of pain experience: the sensory; affective and cognitive. Many of the multiple-item questionnaires of these dimensions are limited by a complexity that diminishes the number of patients who respond. Our high response rate was achieved with simpler numerical rating scales. Future fibromyalgia research would profit from comparison of these single items with more established measures of quality of life [32]. However, similar single-item scales have been found to have acceptable properties in other pain research [21].

It is difficult to compare our results with other interventional treatments since our population is highly selected having failed these "traditional" therapies such as conventional analgesics, antidepressants and physical therapies. Nevertheless, the outcomes appear clinically significant and all the more so for such a selected population.

We collected data on the use of health care resources since overuse of such resources is a reason for referral. In this area there are no established measures. We used GP visits, which we recognise can involve any interaction, even if the original pain problem is addressed. As such, an un- 
changed rate of visiting cannot be easily interpreted, but the result we found, of a reduction in GP visits may be meaningful as it indicates that less GP resources were being utilised. However, this treatment has shortcomings from a health resources perspective. Inpatient stay with monitoring by skilled staff and equipment is required which is expensive. Our costs for an episode of treatment are currently 1800 pounds sterling, which we deliver two to three times per year. This expense is potentially justified by the benefits of this treatment demonstrated here.

The prospective study of adverse effects of this therapy demonstrated that side-effects occur and that all of the potentially serious are cardiovascular. Other than the arrhythmia and pulmonary oedema requiring pharmacological treatment, none required an intervention beyond stopping the infusion. Thus cardiovascular monitoring of ECG and heart rate are prudent to prevent more serious sequelae.

Comparison with other studies of our experience with side-effects is difficult because these have not been quantified before. Edwards [23] reported on 221 patients having intravenous lignocaine therapy up to $5 \mathrm{mg} / \mathrm{kg}$ for a variety of neuropathic pain conditions. Cardiovascular effects were mentioned as a reason to slow or stop the infusion but no further details were given.

Clearly intravenous lignocaine may cause serious adverse effects and although these appear to be uncommon this must be considered against the fact that fibromyalgia itself is not life-threatening. Against this risk must be set the benefits in terms of symptom relief. In our clinical practice we inform patients of our experience with this therapy in terms of its measured benefits and chances of risks, and most patients elect to try it. Notably, those who have gained symptom improvement despite adverse effects request repeated treatment with intravenous lignocaine. Patients may be best placed to balance the risks and benefits of this therapy after being properly informed.

This study supports the benefits previously described with intravenous lignocaine in fibromyalgia. We have been able to quantify the magnitude and duration of effect, which appears to be clinically significant. The side-effects are common and potentially serious and this therapy should be restricted to specialised hospital sites with close surveillance.

There is a need for a larger study of an unselected patient group and for a controlled study of this therapy to determine confidently its effects and separate the pharmacological effects of lignocaine from more indirect effects of treatment such as inpatient hospital stay and bed rest. It is also necessary to compare this treatment with others used for fibromyalgia such as tricyclic antidepressants.

\section{Authors Contributions}

JHR designed the study, analysed the data and wrote up the manuscript. JLS collected the data. GJT analysed the data. GDK revised the manuscript

\section{Competing interests}

None declared.

\section{Acknowledgements}

The authors are grateful to nursing staff of Ward 10, Corbett Hospital for delivering this therapy.

\section{References}

I. Wolfe F, Smythe HA, Yunus MB, et al: The American College of Rheumatology 1990 criteria for the classification of fibromyalgia: Report of the Multicenter Criteria Committee. Arthritis Rheum 1990, 33:160-72

2. Wolfe F, Ross K, Anderson J, Russell IJ, Hebert L: The prevalence and characteristics of fibromyalgia in the general population. Arthritis Rheum 1995, 38:19-27

3. Bennett RM: Fibromyalgia and the disability dilemma: A new era in understanding a complex, multidimensional pain syndrome. Arthritis Rheum 1996, 39:1627-34

4. Ledingham J, Doherty S, Doherty M: Primary fibromyalgia syndrome: An outcome study. $\mathrm{Br}$ J Rheumatol 1993, 32:139-42

5. Goldenberg DC, Felson DT, Dinerman $\mathrm{H}$ : A randomized controlled trial of amitriptyline and naproxen in the treatment of patients with fibromyalgia. Arthritis Rheum 1986, 29:|37|-7

6. Clark S, Tindall E, Bennett RM: A double blind crossover trial of prednisolone versus placebo in the treatment of fibrositis. J Rhematol 1985, I 2:980-3

7. Crofford LJ: Meta-analysis of antidepressants in fibromyalgia. Curr Rheumatol Rep 2001, 3(2): I I 5

8. Jaeschke R, Adachi JD, Guyatt G, Keller J, Wong B: Clinical usefulness of amitriptyline in fibromyalgia: the results of $23 \mathrm{~N}$-of-I randomized controlled trials. J Rheumatol I99I, I 8:447-5 I

9. Burckhardt, Clark SR, Campbell SM, O'Reilly GA, Weins AN, Bennett RM: Multidisciplinary treatment of fibromyalgia. Scan J Rheumatol 1992, 2 I (Suppl):5 I

10. Deluze C, Bosia L, Zirbs A, Chantraine A, Vischer T: Electroacupuncture in fibromyalgia: results of a controlled trial. $\mathrm{Br} M e d$ J 1992, 305:1249-52

II. McCain GA, Bell DA, Mai FM, Halliday PD: A controlled study of the effects of a supervised cardiovascular fitness training program on the manifestations of fibromyalgia. Arthrits Rheum | 988, 3 |: | | 35-4|

12. Verstappen FTJ, van Santen-Hoefft HMS, Bolwijn PH, et al: Effects of a group activity program for fibromyalgia patients on physical fitness and well-being. J Musculoskel Pain 1997, 5:17-28

13. Minor MA, Sanford MK: The role of physical therapy and physical modalities in pain management. Rheum Dic Clin N Am 1999, 25(I):233-48

14. Masi AT: An intuitive person-centred perspective on fibromyalgia syndrome and its management. Balliere's Clin Rheum 1994, 8:957-91

15. Buskila D: Neuroendocrine mechanisms in fibromyalgia chronic fatigue. Best Pract Res Clin Rheumatol 200I, I 5(5):747-58

16. Cohen ML, Quinter JL: Fibromyalgia syndrome: a problem of tautology. Lancet 1993, 342:906-8

17. Kastrup J, Peteren P, Dejgard A, Angelo HR, Hilsted J: Intravenous lidocaine infusion - a new treatment of chronic painful diabetic neuropathy. Pain 1987, 28:69-75

18. Rowbotham MC, Reisner-Keller LA, Fields HL: Both intravenous lidocaine and morphine reduce the pain of postherpetic neuralgia. Neurology 1991, 41: 1024-8

19. Sorenson J, Bengtsson A, Backman E, Henriksson KG, Bengtsson M: Pain analysis in patients with fibromyalgia. Effects of intrave- 
nous morphine, lidocaine and ketamine. Scan J Rheumatol 1995 , 24:360-5

20. Bennett MI, Tai YM: Intravenous lignocaine in the management of primary fibromyalgia syndrome. Int J Clin Pharm Res 1995, 15:115-9

21. Jensen MP, Karoly P, Braver S: The measurement of clinical pain intensity: a comparison of six methods. Pain 1986, 27:1 17-26

22. Jensen MP, Karoly P, Harris P: Assessing the affective component of chronic pain: development of the Pain Discomfort Scale. J Psychosom Res 1991, 35:149-54

23. Edwards WT, Habib F, Burney RG, Begin G: Intravenous lidocaine in the management of various chronic pain states: a review of 2 I I cases. Regional Anaesthesia 1985, I 0:1-6

24. Sorensen J, Graven-Nielsen T, Henriksson KG, Bengtsson M, ArendtNielsen L: Hyperexcitability in fibromyalgia. J Rheumatol 1998, 5:152-5

25. Gibson SJ, Littlejohn GO, Gorman MM, Helme RD, Granges G: Altered heat pain thresholds and cerebral event-related potentials following painful CO2 laser stimulation in subjects with fibromyalgia syndrome. Pain 1994, 58: | 85-93

26. Lorenz J, Grasedyck K, Bromm B: Middle and long latency somatosensory evoked potentials after painful laser stimulation in patients with fibromyalgia syndrome. Electroencephalogr Clin Neurophysiol 1996, 100:165-8

27. Mountz JM, Bradley LA, Modell JG, et al: Fibromyalgia in women. Abnormalities of regional cerebral blood flow in the thalamus and the caudate ucleus are associated with low pain threshold levels. Arthritis Rheum 1995, 38:926-38

28. ladarola MJ, Max MB, Berman KF, et al: Unilateral decrease in thalamic activity observed with positron emission tomography in patients with chronic neuropathic pain. Pain 1995, 63:55-64

29. Jacobson NS, Truax P: Clinical significance: a statistical approach to defining meaningful change in psychotherapy research. J Consult Clin Psychol 1991, 59:12-9

30. Erskine A, Morley S, Pearce S: Memory for pain: a review. Pain 1990, 41:255-65

31. Bryant RA: Memory for pain and affect in chronic pain patients. Pain 1993, 54:347-5।

32. Carr AJ, Thompson PW, Kirwan JR: Quality of life measures. Brit J Rheumatol 1996, 35:275-8।

\section{Pre-publication history}

The pre-publication history for this paper can be accessed here:

http://www.biomedcentral.com/1471-2474/3/21/prepub

Publish with BioMed Central and every scientist can read your work free of charge

"BioMedcentral will be the most significant development for disseminating the results of biomedical research in our lifetime."

$$
\text { Paul Nurse, Director-General, Imperial Cancer Research Fund }
$$

Publish with BMC and your research papers will be:

- available free of charge to the entire biomedical community

- peer reviewed and published immediately upon acceptance

- cited in PubMed and archived on PubMed Central

- yours - you keep the copyright

Submit your manuscript here:

http://www.biomedcentral.com/manuscript/
BioMedcentral.com editorial@biomedcentral.com 